\title{
Efficacy and safety of drug-eluting stents in elderly patients: A meta-analysis of randomized trials
}

\author{
SungA Bae ${ }^{1, *}$, Yongcheol Kim ${ }^{1, *}$, Bill D. Gogas ${ }^{2}$, Min Chul Kim ${ }^{1}$, Doo Sun Sim ${ }^{1}$, \\ Young Joon Hong ${ }^{1}$, Ju Han Kim ${ }^{1}$, Youngkeun $\mathrm{Ahn}^{1}$, Myung Ho Jeong ${ }^{1}$ \\ ${ }^{1}$ Department of Cardiology, Chonnam National University Hospital, Gwangju, Korea \\ ${ }^{2}$ The Spencer B. King III Catheterization Laboratory, Nanjing First Hospital, \\ Nanjing Medical University, Nanjing, China
}

\begin{abstract}
Background: Current guidelines recommend newer generation drug-eluting stents (DES) over bare-metal stents (BMS) in patients with ischemic heart disease. However, there is no age-specific recommendation in elderly patients.

Methods: Meta-analysis was performed of 6 randomized studies enrolling 5,042 elderly patients who underwent percutaneous coronary intervention (PCI) with stent implantation (DES, $n=2,579$; BMS, $n=2,463)$.

Results: Combined data indicated a significant reduction in major adverse cardiovascular events (MACEs) with use of DES (odds ratio [OR] 0.56, 95\% confidence interval [CI] 0.44-0.71, $p<0.001$ ). Moreover, use of DES was associated with a significantly lower incidence of myocardial infarction (OR 0.54, 95\% CI 0.36-0.81, $p=0.003)$ and repeat revascularization (OR 0.44, 95\% CI 0.31-0.62, $p<0.001$ ), was compared to that with the use of BMS. Stent thrombosis and bleeding complication rates were not significantly different between groups. In a subgroup meta-analysis, short duration (1 or 6 months) dual antiplatelet therapy (DAPT) was associated with a significantly lower MACE rate (OR 0.49, 95\% CI 0.34-0.80; $p=0.003)$ in elderly patients who underwent PCI with everolimuseluting stent implantation, compared with that using long duration DAPT.

Conclusions: This meta-analysis provides clinically relevant evidence that DES rather than BMS should be selected for elderly patients. (Cardiol J 2021; 28, 2: 223-234)

Key words: drug-eluting stent, bare-metal stent, elderly, clinical trials, clinical research
\end{abstract}

\section{Introduction}

The introduction of drug-eluting stents (DES) and advanced pharmacotherapy resulted in a significant reduction in restenosis rates [1-5]. This improvement, however, increased the prevalence of bleeding complications due to use of DES and longer duration of dual antiplatelet therapy (DAPT), compared to that using bare-metal stents
(BMS) [6]. Long duration of DAPT after DES deployment was associated with higher risk of major bleeding complications despite the beneficial effects of novel platforms, especially in vulnerable populations such as patients over 75 years old [7].

Until recently, guidelines have not provided evidence-based recommendations for treatment of elderly patients [8]. Recently, the SYNERGY II Everolimus eluting stent in patients older than

Address for correspondence: Dr. Myung Ho Jeong, MD, PhD, FACC, FAHA, FESC, FSCAI, FAPSIC, Principal Investigator of Korea Acute Myocardial Infarction Registry, Heart Research Center, Chonnam National University Hospital, 671 Jaebongro, Dong-gu, Gwangju, 61469, Republic of Korea, tel: +82-62-220-6243, fax: +82-62-228-7174, e-mail: myungho@chollian.net

Received: 5.08.2019 Accepted: 29.10.2019

*These authors equally contributed to this work as the co-first authors.

This article is available in open access under Creative Common Attribution-Non-Commercial-No Derivatives 4.0 International (CC BY-NC-ND 4.0) license, allowing to download articles and share them with others as long as they credit the authors and the publisher, but without permission to change them in any way or use them commercially. 
75 years, undergoing coronary revascularization associated with a short DAPT (SENIOR) trial demonstrated that use of DES rather than BMS in patients older than 75 years results in lower adverse clinical event rates at 1 year [9]. These observations were also previously seen in the Xience or Vision Stents for the Management of Angina in the Elderly (XIMA) trial, which demonstrated a reduction in myocardial infarction (MI) and in-stent restenosis in the DES group without an increase in bleeding [10]. The superiority of DES in the SENIOR trial was mainly due to a reduction of target lesion revascularization (TLR), but there were no significant differences between all-cause death, MI, and stroke. Therefore, it is unclear whether the clinical benefits of DES were overestimated [11]. Herein, a meta-analysis was performed of randomized studies aiming to assess the benefits and risks associated with DES vs. BMS use for percutaneous coronary intervention (PCI) in elderly patients.

\section{Methods}

This study was designed in accordance with the Preferred Reporting Items for Systematic reviews and Meta-Analyses (PRISMA) guidelines [12]. A comprehensive MEDLINE, EMBASE, and Cochrane database search was conducted until September 6, 2018, using the following medical subject headings alone and in different combinations: "drug-eluting stent(s)", "DES", "bare-metal stent(s)", "BMS", "coronary artery disease" and "elderly patients". Randomized studies that evaluated elderly patients undergoing PCI and reported on clinical outcomes with follow-up time $\geq 12$ months were included. Conventionally, "elderly" has been defined as a chronological age of $\geq 65$ years. In the present study however, elderly patients were defined as $>70$ years old. Only full articles in peer-reviewed journals were considered.

Two investigators (SAB, YK) extracted baseline study characteristics, clinical outcomes, and DAPT duration of interest from the retrieved studies. Any divergences were resolved by consensus. The number of events associated with clinical outcomes was tabulated for the longest follow-up available.

The primary endpoint was major adverse cardiovascular events (MACEs), defined as a composite of cardiac death, MI, and repeat revascularization, including TLR and target vessel revascularization (TVR). Secondary endpoints were individual components of MACE, definite/probable stent thrombosis, as defined by the Academic Research Consortium, and bleeding complications according to both Thrombolysis in Myocardial Infarction and Bleeding Academic Research Consortium classifications $[13,14]$. Subgroup meta-analysis of DES implantation with short (1 or 6 months) vs. long (> 12 months) DAPT duration was performed to determine MACE, stent thrombosis, and bleeding complication rates. Moreover, a meta-regression analysis was performed to identify moderators in a linear relationship among baseline characteristics according to the percentage of hypertension, diabetes mellitus, dyslipidemia, and acute coronary syndrome (ACS). The SENIOR trial was included in the short DAPT group, while the XIMA, Basel Stent Kosten Effektivitäts Trial-PROspective Validation Examination (BASKET-PROVE), and Everolimus-Eluting Stents Versus Bare-Metal Stents in ST-Segment Elevation Myocardial Infarction (EXAMINATION) trials were included in the long DAPT group.

Quality assessment was performed for both study groups. The risk of bias was assessed of each study with the Cochrane tool and the Grading of Recommendations, Assessment, Development and Evaluation (GRADE) tool [15, 16] was used to assess quality as high, moderate, low, or very low. Most clinical trials showed low evidence of bias with the Cochrane tool. In addition, the level of evidence was strong for primary outcomes assessed with the GRADE tool.

\section{Statistical analysis}

The number of patients, events, means, standard deviations (SDs), and percentages were abstracted. Estimates were calculated with a random effects model and confirmed with a fixed effects model and was expressed as odds ratios (ORs). A p-value $\leq 0.05$ (2-tailed) indicated statistical significance. The random effects model was prioritized over the fixed effects model and sensitivity analysis was conducted to identify sources of inconsistency. The $\mathrm{I}^{2}$ statistic was used for evaluation of heterogeneity between studies with values of $<30 \%, 30 \%$ to $60 \%$, and $>60 \%$, corresponding to low, moderate, and high degrees of heterogeneity, respectively [17]. Publication bias was assessed using both the Egger and Begg's tests. A p-value $<0.05$ indicated evidence of bias [18]. All data analyses were performed using $\mathrm{R}$ version 3.5.0 (R Foundation for Statistical Computing, Vienna, Austria). This study was registered with PROSPERO, number CRD42019112969. 


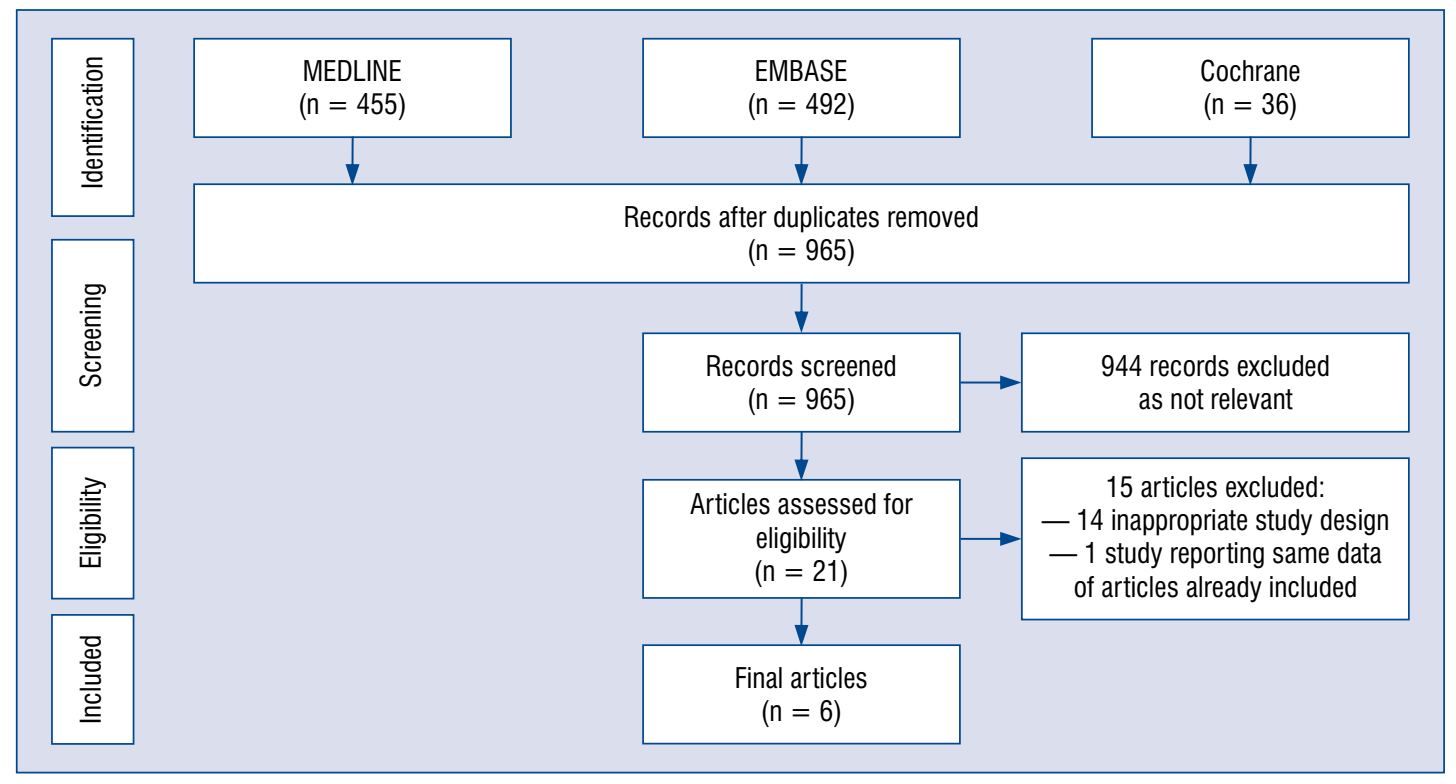

Figure 1. Preferred Reporting Items for Systematic reviews and Meta-Analyses (PRISMA) flow chart for the trial selection process.

\section{Results}

The flow chart of the study selection process is shown in Figure 1. Six multi-center randomized controlled trials enrolling 5,042 elderly patients with coronary artery disease (CAD), who underwent PCI with either DES ( $\mathrm{n}=2,579)$ or BMS $(\mathrm{n}=2,463)$ implantation were included $[9,10$, 19-22]. The study design and characteristics of the trials involved are shown in Table 1. When studies reported results from both unmatched and matched populations, data regarding the matched subgroup were considered. The mean follow-up completion for all trials was relevant, with an overall rate of $98 \%$. The recommended DAPT duration varied between trials (1-12 months), but was the same in both the DES and BMS groups, except in the XIMA trial (1 month of DAPT for patients receiving BMS and 12 months for patients receiving DES).

During long-term follow-up (range 1-2 years), combined data indicated a significant reduction in MACE with DES use (OR 0.56, 95\% confidence interval [CI] 0.44-0.71, $\mathrm{p}<0.001$, Fig. 2A). There was no significant difference in stent thrombosis between groups (OR 0.68, 95\% CI0.40-1.14, p = 0.142, Fig. 2B). Bleeding complication rates were similar for both groups (OR 0.96, 95\% CI 0.78-1.18, p = 0.686, Fig. $2 \mathrm{C}$ ). In addition, the risk of cardiac death did not differ between the groups (OR 0.81, 95\% CI 0.65-1.02, $p=0.075$, Fig. $3 \mathrm{~A}$ ). However, use of DES rather than BMS was associated with a significantly lower inci- dence of MI (OR 0.54, 95\% CI 0.36-0.81, $\mathrm{p}=0.003$, Fig. 3B) and repeat revascularization (OR $0.44,95 \%$ CI $0.31-0.62, \mathrm{p}<0.001$, Fig. 3C). The funnel plots and the Egger and Begg tests did not suggest any significant publication bias (Fig. 4).

In elderly patients who underwent PCI with everolimus-eluting stent (EES) implantation, subgroup meta-analysis showed a significant decrease in MACE in the short DAPT (1 or 6 months) group (OR 0.49, 95\% CI 0.34-0.80; $\mathrm{p}=0.003$ ), without statistical heterogeneity $\left(\mathrm{I}^{2}=23.7 \%\right.$; $\mathrm{p}=0.08$; Fig. $5 \mathrm{~A}$ ). However, there were no significant differences in stent thrombosis and bleeding complication rates according to DAPT duration (Fig. 5B, C). Subgroup analysis showed a significant decrease in MACE with all DES types, including EESs, biolimus-eluting stents, and zotarolimuseluting stents (ZESs). Moreover, use of a ZES was associated with a significantly lower incidence of definite/probable stent thrombosis (OR 0.40, 95\% CI 0.20-0.83; Fig. 6).

\section{Discussion}

The main findings of the present study were as follows: 1) DES deployment was associated with significant reduction in MACE, MI, and repeat revascularization in elderly patients; 2 ) DES implantation was associated with the risk of stent thrombosis and bleeding complications similar to that of BMS implantation; 3) In subgroup meta- 


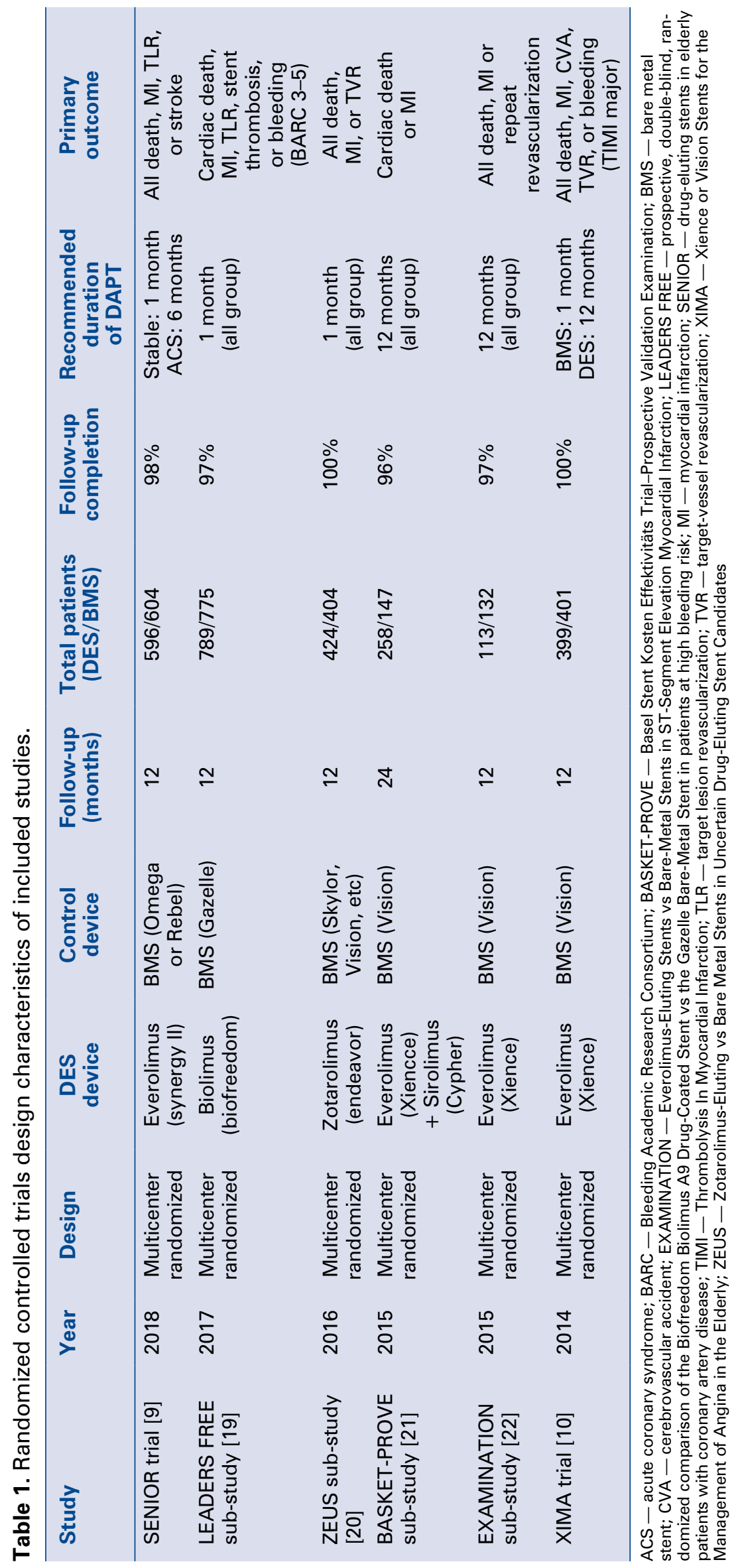




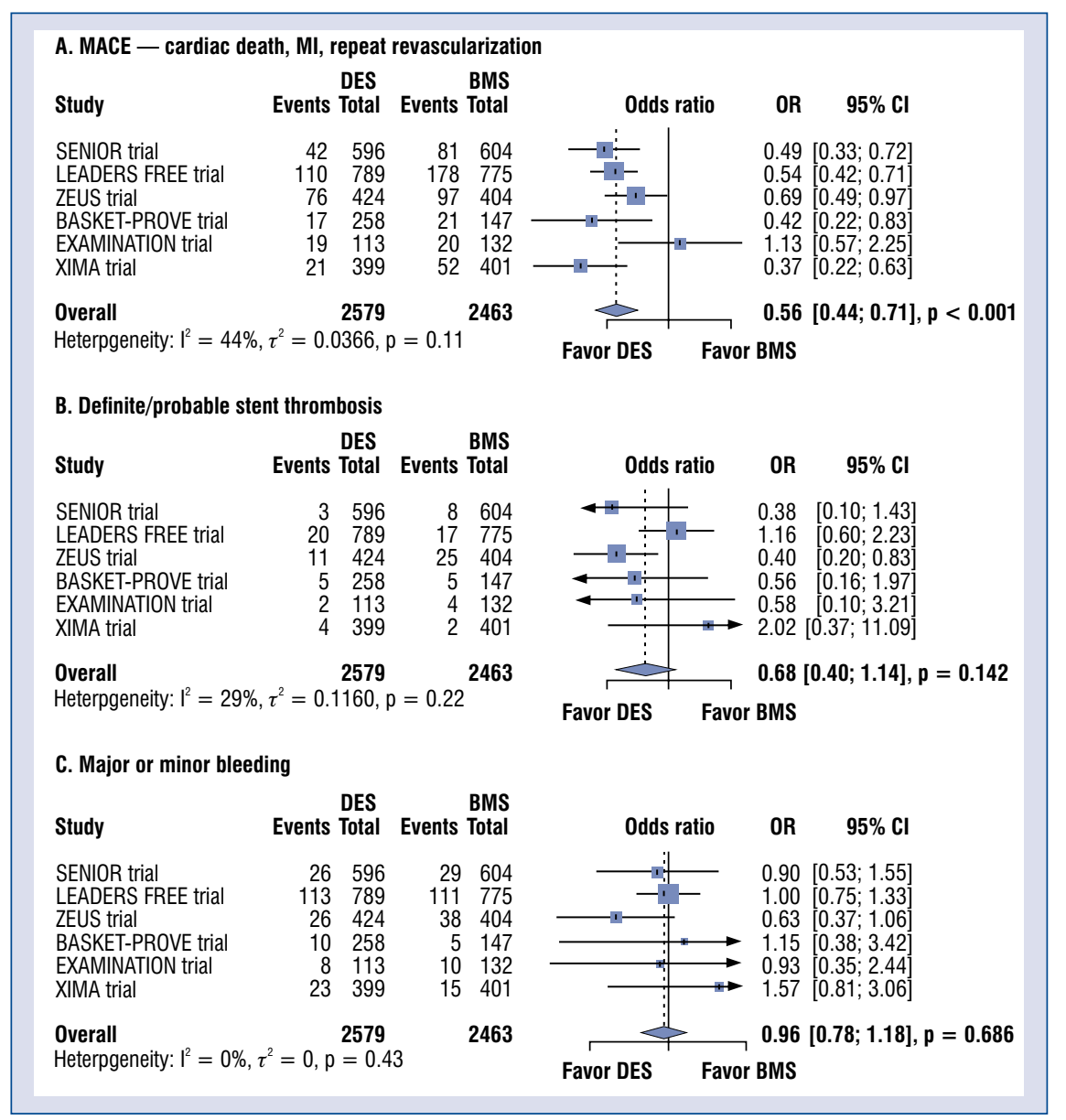

Figure 2. Forest plot for the risk of major adverse cardiovascular event (MACE; A), definite/probable stent thrombosis (B), and bleeding (C) in elderly patients treated with drug-eluting stents (DES) versus bare-metal stents (BMS); $\mathrm{MI}$ - myocardial infarction; $\mathrm{Cl}$ - confidencial interval.

analysis, clinical outcomes were similar for short and long DAPT duration in elderly patients who underwent PCI with EES implantation.

Current guidelines recommend stenting with the newer generation of DES rather than BMS in patients with ischemic heart disease including ST-segment elevation myocardial infarction (STEMI), because of better efficacy and safety profiles [23, 24]. Moreover, guidelines support DES as the preferred treatment option regardless of DAPT duration in patients with high bleeding risk [25]. Nevertheless, age-specific recommendations in elderly patients are not available; thus, BMS has been the preferred option in elderly patients due to shorter DAPT duration [8].

As shown in Table 2, the results of 6 randomized trials, including 4 studies involving patients older than 80 years of age on average, can be seen as appropriate evidence to determine PCI strategy in elderly patients. However, differences in the definitions of primary and secondary outcomes make it difficult to comprehensively assess the benefits of DES in the treatment of elderly patients. The beneficial effects of DES on all-cause death have only been reported in a sub-study of the BASKET-PROVE trial [21]. Furthermore, the cardiac death rate was comparable to that in 6 of the studies included. In contrast to the other 4 randomized studies, the SENIOR trial and substudy of the EXAMINATION trial did not show a difference in the risk of MI in both the DES and BMS groups [9, 22]. Particularly in the sub-study of the EXAMINATION trial for STEMI patients, DES use did not show any benefits over BMS use in patients over 75 years old [22]. In meta-analysis, DES use was associated with a significant reduction in redefined MACE, including cardiac death, MI, and repeat revascularization. Except for the sub-study of the EXAMINATION trial, the studies included showed benefits of DES use for MACE in 


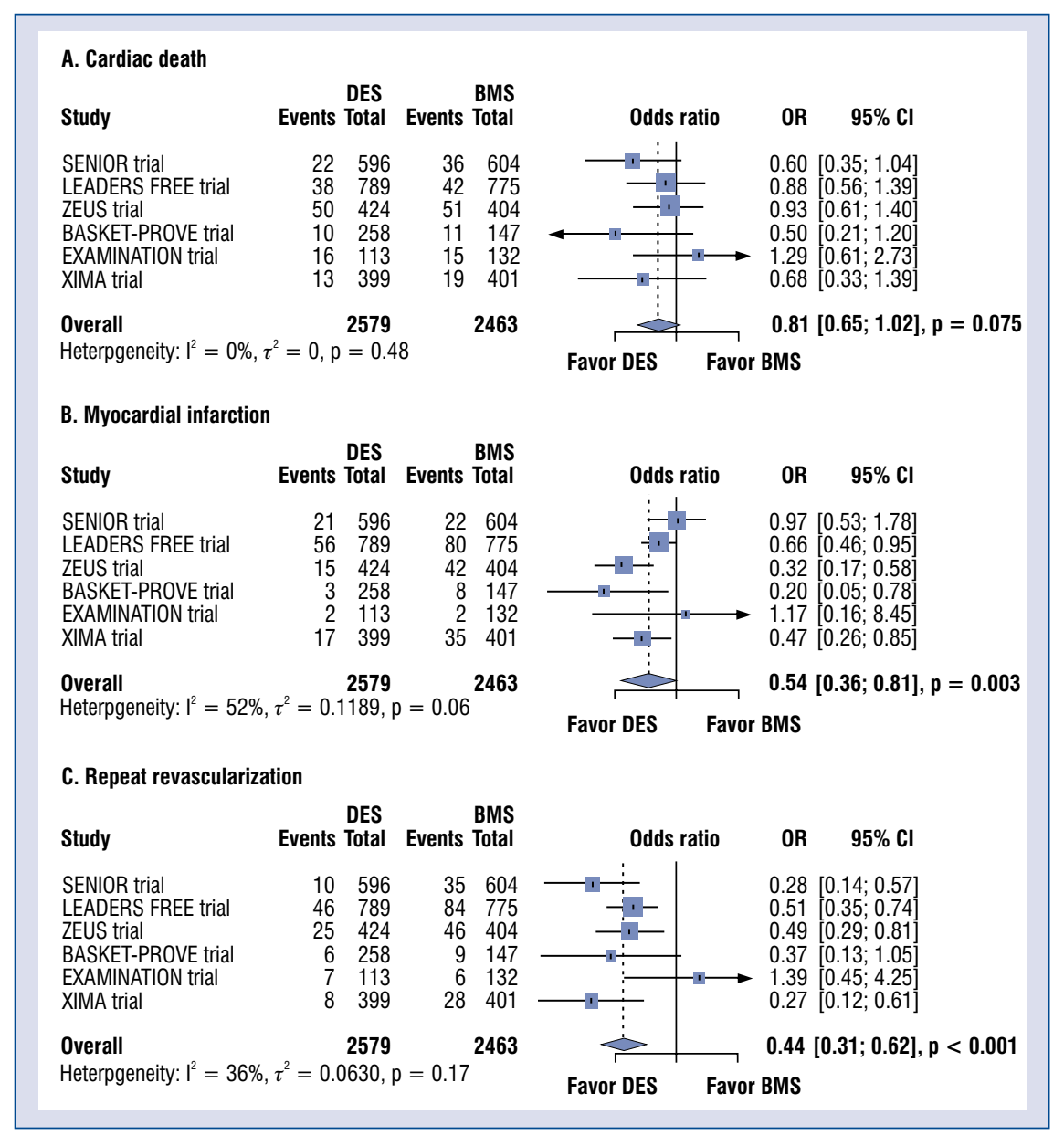

Figure 3. Forest plot for the risk of cardiac death (A), myocardial infarction (B), and repeat revascularization (C) in elderly patients treated with drug-eluting stents (DES) versus bare-metal stents (BMS); $\mathrm{Cl}$ — confidencial interval.

elderly patients. Furthermore, our pooled analysis demonstrated that PCI with DES implantation was apparently superior to BMS use in terms of MI and repeat revascularization. The Norwegian Coronary Stent Trial (NORSTENT), a large randomized trial comparing long-term outcomes after DES $(\mathrm{n}=4,504)$ vs. BMS use $(\mathrm{n}=4,509)$, reported results similar to those in the present study, with a significantly lower rate of repeat revascularization at 6 years in the group receiving DES [26]. However, NORSTENT enrolled relatively younger patients, and did not show the benefits of DES use for MI compared with the findings in the present study. Although it is difficult to compare the outcomes of MI between the NORSTENT and the present study, the differences may reflect the significant benefit of DES for elderly patients who tend to have more extensive and complex lesions. The risk of stent thrombosis and bleeding complications with use of DES was comparable to that of BMS in the 6 trials included and the NORSTENT. This tendency was also observed in the meta-analysis. Therefore, when considering efficacy and safety, DES use should be considered in elderly patients, as described in the current guidelines.

The scoring systems used to determine DAPT duration include the DAPT score and PREdicting bleeding Complications in patients undergoing Stent implantation and subsequent Dual Anti Platelet Therapy (PRECISE-DAPT) [27, 28]. In both scoring models, age has been used to assess bleeding and ischemic risk since post-PCI bleeding complications were associated with a significant increase in adverse clinical outcomes in patients older than 75 years of age [29]. However, the usefulness of these scores for improving outcomes remains unclear, due to the lack of evidence in the setting of randomized controlled trials. According to current guidelines, short DAPT duration should be considered in patients with high bleeding risk 


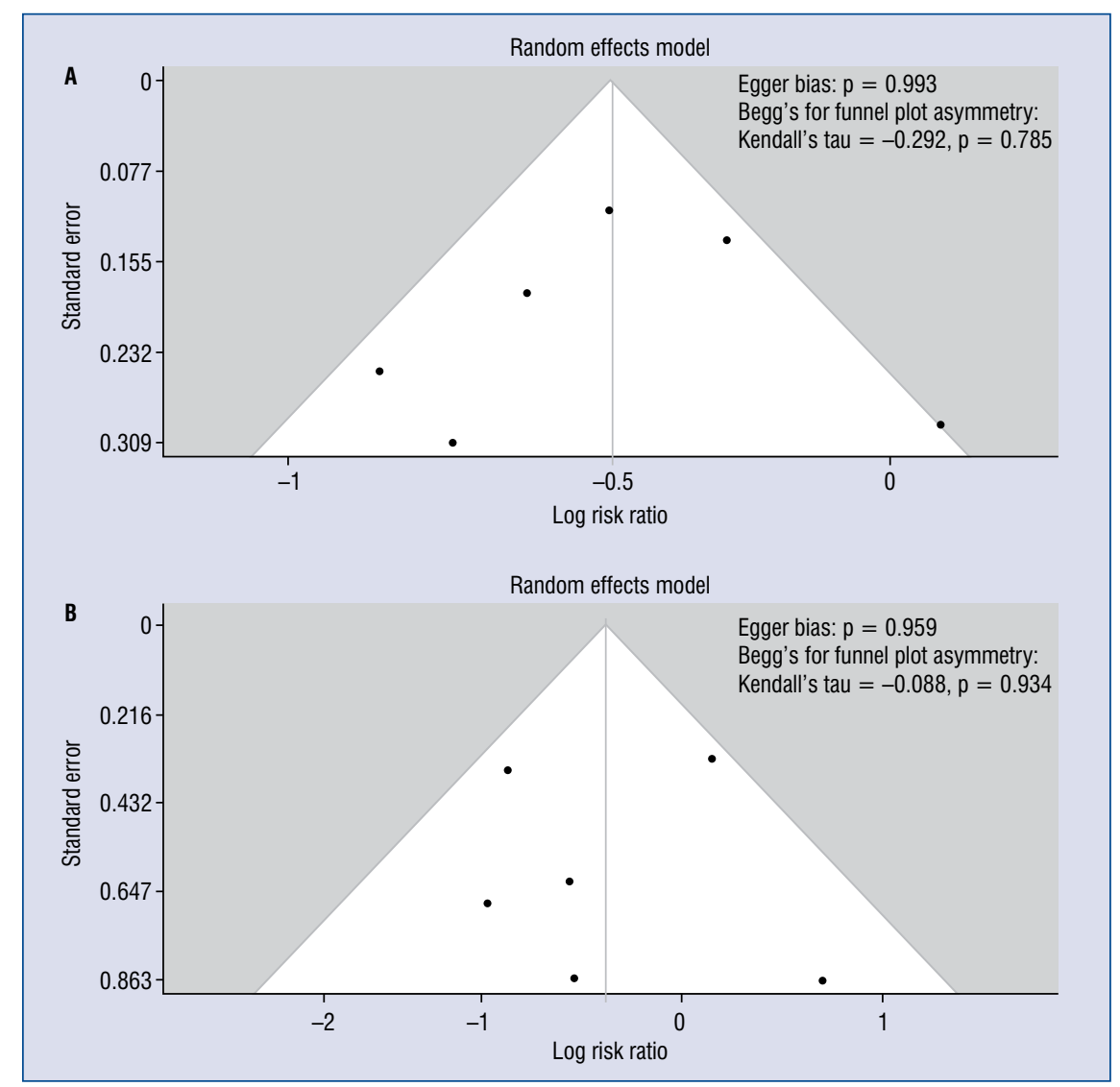

Figure 4. Funnel plot. A. Funnel plot for major adverse cardiovascular events (MACE); B. Funnel plot for definite/ /probable stent thrombosis.

(PRECISE-DAPT score $\geq 25$ ), with 3 months of DAPT for stable CAD and 6 months of DAPT for ACS [25]. The current subgroup meta-analysis in elderly patients who underwent PCI with EES implantation showed no significant differences between use of short DAPT duration and long DAPT duration in stent thrombosis and bleeding complications, as shown in Figure 5. Moreover, short DAPT duration was associated with a significantly lower incidence of MACE, compared with using long DAPT duration. Therefore, short DAPT duration is as safe as long DAPT duration in elderly patients who undergo PCI with EES implantation.

\section{Limitations of the study}

There were several limitations in this study. First, there was considerable heterogeneity between studies, which was particularly evident when comparing studies using different designs. Second, the definition of MACE was different in each study. Therefore, MACE was redefined to reduce confounders. Third, the definition of elderly varies from 65 to 75 years of age, but the present study defined elderly to be $>70$ years of age, since there have been few randomized controlled trials in those aged $\geq 75$ years. Fourth, differences in DAPT duration according to DES or BMS use were reported in only 1 of the 6 trials included (XIMA trial: 1 month of DAPT for patients receiving BMS and 12 months for those receiving DES), which could affect outcomes. Fifth, there are two types of EES, durable polymer EES (XIENCE ${ }^{\mathrm{TM}}$, Abbott Vascular, Santa Clara, CA, USA) and bioabsorbable polymer EES (SYNERGY ${ }^{\mathrm{TM}}$, Boston Scientific, Marlborough, MA, USA). Bioabsorbable polymer (BP)-DES implantation was reported to have better endothelial healing and conjugate protein expression than durable polymer-DES implantation [30]. Unique characteristics of BP-DES might affect the results of short and long DAPT duration on subgroup analysis. However, subgroup analysis included bioabsorbable or durable polymer EES. In addition, the proportion of ACS patients could not be assessed in the short and long DAPT duration groups and the comparison of DAPT duration was not randomly allocated between studies. Thus, a careful interpretation of subgroup analysis of DAPT duration is necessary. 


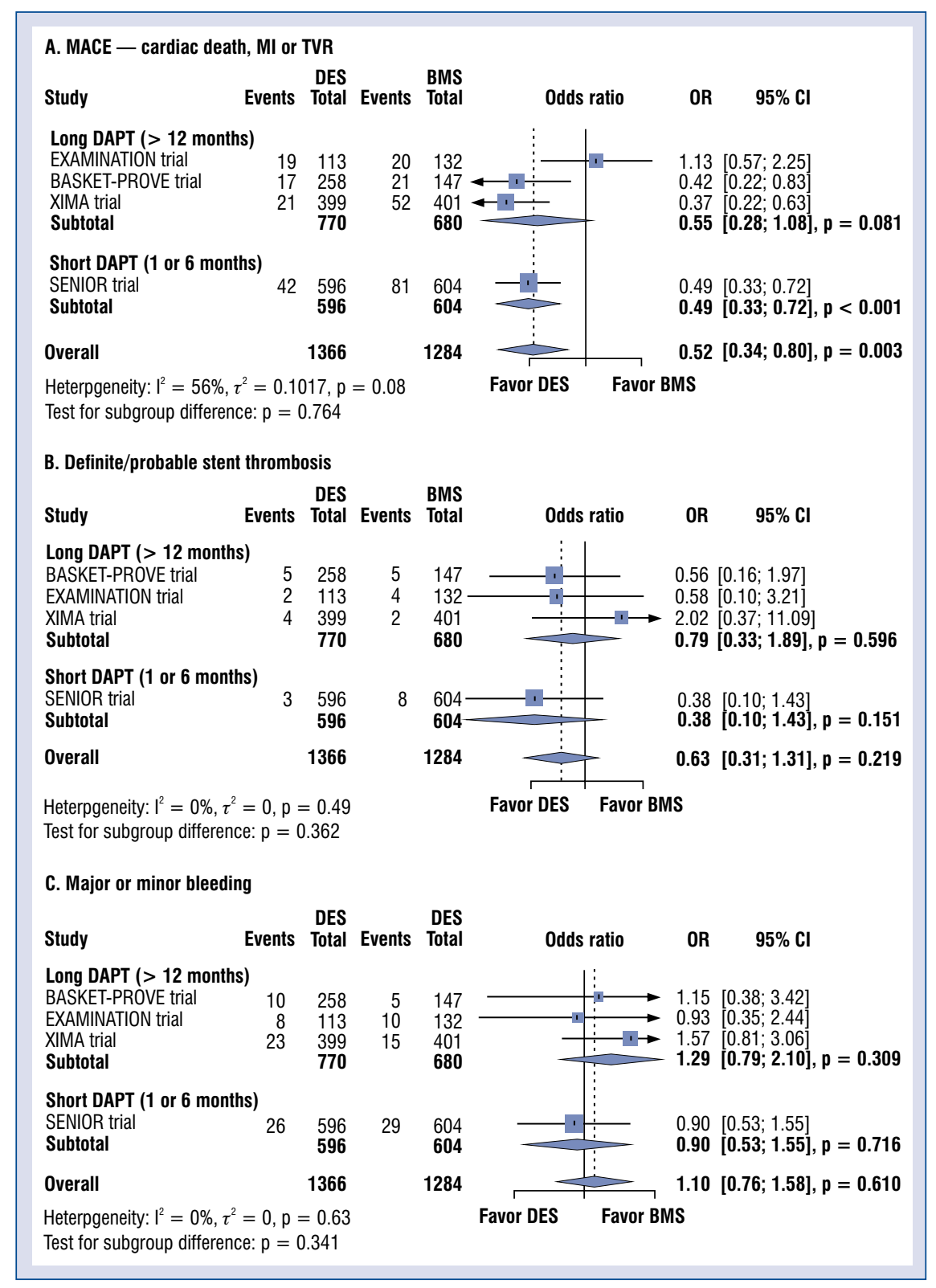

Figure 5. A-C. Subgroup meta-analysis of the effect of short $(\leq 1$ or 6 months) versus long ( $>12$ months) dual antiplatelet therapy (DAPT) duration in elderly patients who underwent percutaneous coronary intervention (PCI) with everolimus-eluting stent (EES) implantation. DES — drug-eluting stents; BMS — bare-metal stents; $\mathrm{Cl}$ - confidence interval; MACE - major adverse cardiovascular events; MI - myocardial infarction; TVR - target vessel revascularization.

\section{Conclusions}

This meta-analysis builds upon recent evidence to support the efficacy and safety of DES use, and provides clinically relevant evidence that DES rather than BMS should be selected for treatment of elderly patients. Furthermore, short DAPT duration should be considered when PCI with EES implantation is performed in elderly patients.

\section{Acknowledgements}

This study was supported by a grant of Chonnam National University Hospital Biomedical Research Institute (BCRI18015), by a grant of the Korean Health Technology R \& D Project (HI13C1527), sponsored by the Ministry of Health and Welfare, Republic of Korea.

Conflict of interest: None declared 


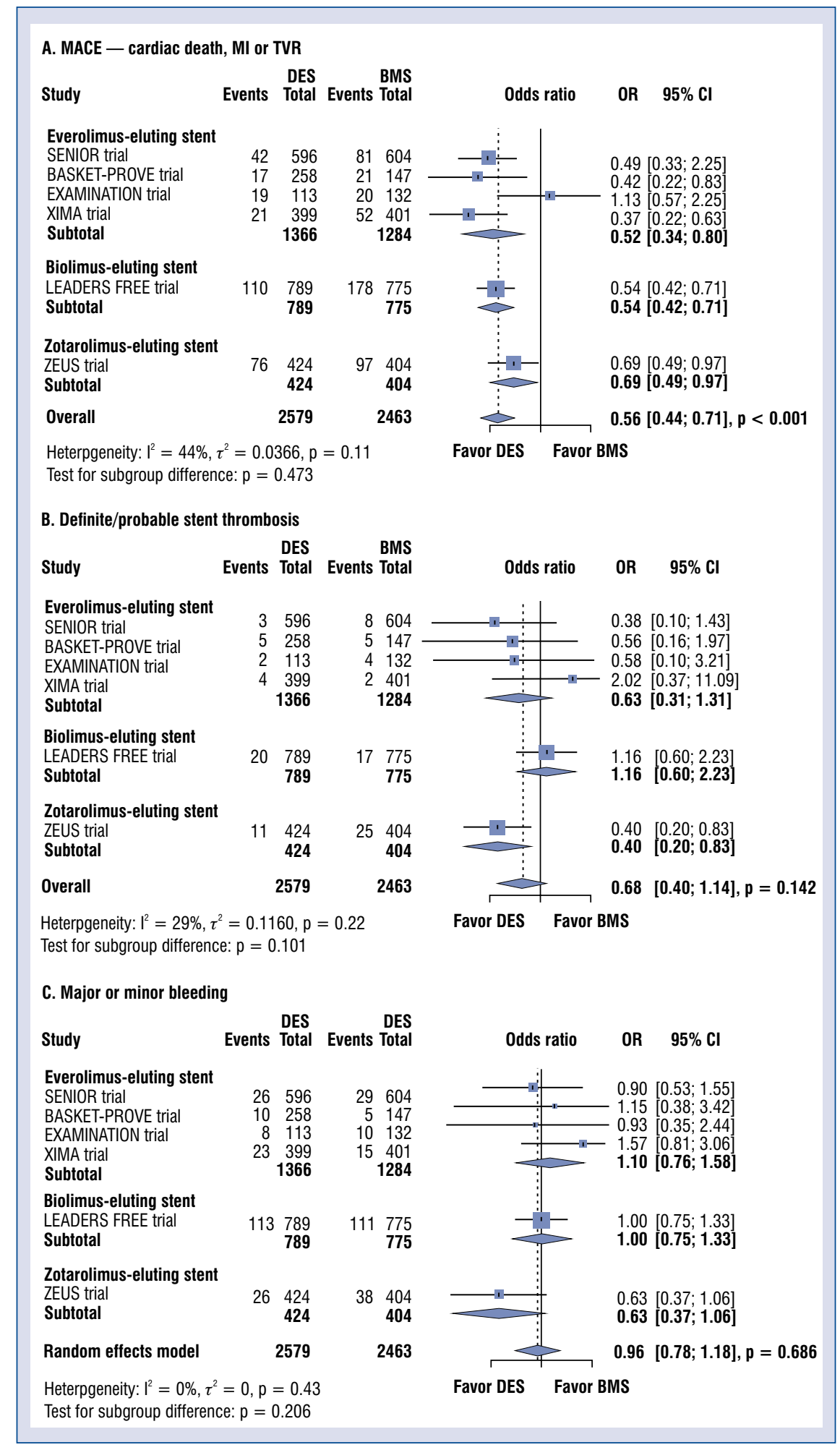

Figure 6. A-C. Subgroup meta-analysis of the effect according to drug-eluting stents (DES) type. BMS - bare-metal stents; MACE - major adverse cardiovascular events; MI — myocardial infarction; TVR — target vessel revascularization; $\mathrm{Cl}$ - confidence interval. 


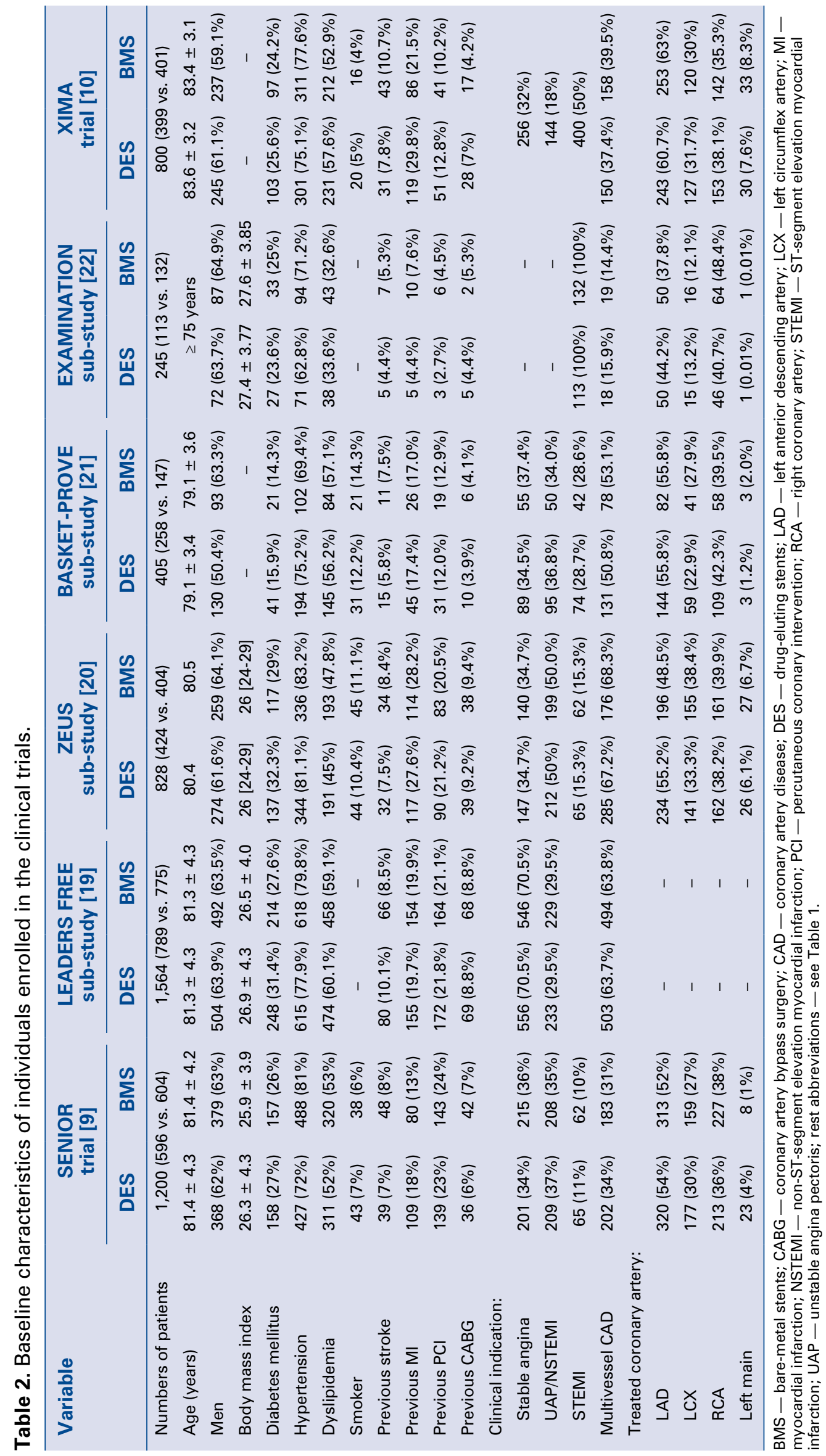




\section{References}

1. Menichelli M, Parma A, Pucci E, et al. Randomized trial of Sirolimus-Eluting Stent Versus Bare-Metal Stent in Acute Myocardial Infarction (SESAMI). J Am Coll Cardiol. 2007; 49(19): 1924-1930, doi: 10.1016/j.jacc.2007.01.081, indexed in Pubmed: 17498576.

2. Spaulding C, Henry P, Teiger E, et al. TYPHOON Investigators. Sirolimus-eluting versus uncoated stents in acute myocardial infarction. N Engl J Med. 2006; 355(11): 1093-1104, doi: 10.1056/ NEJMoa062006, indexed in Pubmed: 16971716.

3. Laarman GJ, Suttorp MJ, Dirksen MT, et al. Paclitaxel-eluting versus uncoated stents in primary percutaneous coronary intervention. N Engl J Med. 2006; 355(11): 1105-1113, doi: 10.1056/ NEJMoa062598, indexed in Pubmed: 16971717.

4. Stettler C, Wandel S, Allemann S, et al. Outcomes associated with drug-eluting and bare-metal stents: a collaborative network meta-analysis. Lancet. 2007; 370(9591): 937-948, doi: 10.1016/ S0140-6736(07)61444-5, indexed in Pubmed: 17869634.

5. Horst B, Rihal CS, Holmes DR, et al. Comparison of drugeluting and bare-metal stents for stable coronary artery disease. JACC Cardiovasc Interv. 2009; 2(4): 321-328, doi: 10.1016/j. jcin.2008.11.010, indexed in Pubmed: 19463444.

6. Cassese S, Byrne RA, Tada T, et al. Clinical impact of extended dual antiplatelet therapy after percutaneous coronary interventions in the drug-eluting stent era: a meta-analysis of randomized trials. Eur Heart J. 2012; 33(24): 3078-3087, doi: 10.1093/eurheartj/ehs318, indexed in Pubmed: 23091199.

7. Gerber RT, Arri SS, Mohamed MO, et al. Age is not a bar to PCI: Insights from the long-term outcomes from off-site PCI in a real-world setting. J Interv Cardiol. 2017; 30(4): 347-355, doi: 10.1111/joic.12400, indexed in Pubmed: 28670721.

8. Rich MW, Chyun DA, Skolnick AH, et al. Knowledge Gaps in Cardiovascular Care of the Older Adult Population: A Scientific Statement From the American Heart Association, American College of Cardiology, and American Geriatrics Society. J Am Coll Cardiol. 2016; 67(20): 2419-2440, doi: 10.1016/j. jacc.2016.03.004, indexed in Pubmed: 27079335.

9. Varenne O, Cook S, Sideris G, et al. Drug-eluting stents in elderly patients with coronary artery disease (SENIOR): a randomised single-blind trial. Lancet. 2018; 391(10115): 41-50, doi: 10.1016/S0140-6736(17)32713-7, indexed in Pubmed: 29102362.

10. de Belder A, de la Torre Hernandez JM, Lopez-Palop R, et al. A prospective randomized trial of everolimus-eluting stents versus bare-metal stents in octogenarians: the XIMA Trial (Xience or Vision Stents for the Management of Angina in the Elderly). J Am Coll Cardiol. 2014; 63(14): 1371-1375, doi: 10.1016/j. jacc.2013.10.053, indexed in Pubmed: 24216285.

11. Leidi F, Schiavon R. Drug-eluting stents vs. bare metal stents in elderly patients. Intern Emerg Med. 2018; 13(5): 773-774, doi: 10.1007/s11739-018-1839-5, indexed in Pubmed: 29594816.

12. Moher D, Liberati A, Tetzlaff J, et al. Preferred reporting items for systematic reviews and meta-analyses: the PRISMA statement. BMJ. 2009; 339: b2535-b2535, doi: 10.1136/bmj.b2535.

13. Cutlip DE, Windecker S, Mehran R, et al. Academic Research Consortium. Clinical end points in coronary stent trials: a case for standardized definitions. Circulation. 2007; 115(17): 23442351, doi: 10.1161/CIRCULATIONAHA.106.685313, indexed in Pubmed: 17470709.

14. Mehran R, Rao SV, Bhatt DL, et al. Standardized bleeding definitions for cardiovascular clinical trials: a consensus report from the Bleeding Academic Research Consortium. Circulation. 2011; 123(23): 2736-2747, doi: 10.1161/CIRCULATIONAHA.110.009449, indexed in Pubmed: 21670242.

15. Higgins JPT, Altman DG, Gøtzsche PC, et al. The Cochrane Collaboration's tool for assessing risk of bias in randomised trials. BMJ. 2011; 343: d5928, doi: 10.1136/bmj.d5928, indexed in Pubmed: 22008217.

16. Atkins D, Best D, Briss PA, et al. Grading quality of evidence and strength of recommendations. BMJ. 2004; 328(7454): 1490, doi: 10.1136/bmj.328.7454.1490, indexed in Pubmed: 15205295.

17. Higgins JPT, Thompson SG, Deeks JJ, et al. Measuring inconsistency in meta-analyses. BMJ. 2003; 327(7414): 557-560, doi: 10.1136/bmj.327.7414.557, indexed in Pubmed: 12958120.

18. Egger M, Davey Smith G, Schneider M, et al. Bias in meta-analysis detected by a simple, graphical test. BMJ. 1997; 315(7109): 629-634, doi: 10.1136/bmj.315.7109.629, indexed in Pubmed: 9310563.

19. Morice MC, Talwar S, Gaemperli O, et al. Drug-coated versus bare-metal stents for elderly patients: A predefined sub-study of the LEADERS FREE trial. Int J Cardiol. 2017; 243: 110-115, doi: 10.1016/j.ijcard.2017.04.079, indexed in Pubmed: 28579168.

20. Ariotti S, Adamo M, Costa F, et al. Is Bare-Metal Stent Implantation Still Justifiable in High Bleeding Risk Patients Undergoing Percutaneous Coronary Intervention?: A Pre-Specified Analysis From the ZEUS Trial. JACC Cardiovasc Interv. 2016; 9(5): 426-436, doi: 10.1016/.j.jin.2015.11.015, indexed in Pubmed: 26965932.

21. Kurz DJ, Bernheim AM, Tüller D, et al. Improved outcomes of elderly patients treated with drug-eluting versus bare metal stents in large coronary arteries: results from the BAsel Stent Kosten-Effektivitäts Trial PROspective Validation Examination randomized trial. Am Heart J. 2015; 170(4): 787-795.e1, doi: 10.1016/j.ahj.2015.07.009, indexed in Pubmed: 26386803.

22. Ielasi A, Brugaletta S, Silvestro A, et al. Everolimus-eluting stent versus bare-metal stent in elderly ( $\geq 75$ years) versus nonelderly ( $<75$ years) patients with ST-elevation myocardial infarction undergoing primary percutaneous coronary intervention: insights from the examination trial. Int J Cardiol. 2015; 179: 73-78, doi: 10.1016/j.ijcard.2014.10.038, indexed in Pubmed: 25464418.

23. Ibanez B, James S, Agewall S, et al. 2017 ESC Guidelines for the management of acute myocardial infarction in patients presenting with ST-segment elevation: The Task Force for the management of acute myocardial infarction in patients presenting with ST-segment elevation of the European Society of Cardiology (ESC). Eur Heart J. 2018; 39(2): 119-177, doi: 10.1093/eurheartj/ ehx393, indexed in Pubmed: 28886621.

24. Neumann FJ, Sousa-Uva M, Ahlsson A, et al. Considerations for the choice between coronary artery bypass grafting and percutaneous coronary intervention as revascularization strategies in major categories of patients with stable multivessel coronary artery disease: an accompanying article of the task force of the $2018 \mathrm{ESC} / \mathrm{EACTS}$ guidelines on myocardial revascularization. Eur Heart J. 2019; 40(2): 204-212, doi: 10.1093/eurheartj/ ehy532, indexed in Pubmed: 30165435.

25. Valgimigli M, Bueno H, Byrne RA, et al. 2017 ESC focused update on dual antiplatelet therapy in coronary artery disease developed in collaboration with EACTS: The Task Force for dual antiplatelet therapy in coronary artery disease of the European Society of Cardiology (ESC) and of the European Association for Cardio-Thoracic Surgery (EACTS). Eur Heart J. 2018; 39(3): 213-260, doi: 10.1093/eurheartj/ehx419, indexed in Pubmed: 28886622. 
26. Bønaa KH, Mannsverk J, Wiseth R, et al. Drug-eluting or baremetal stents for coronary artery disease. N Engl J Med. 2016; 375(13): 1242-1252, doi: 10.1056/NEJMoa1607991, indexed in Pubmed: 27572953.

27. Yeh RW, Secemsky EA, Kereiakes DJ, et al. Development and Validation of a Prediction Rule for Benefit and Harm of Dual Antiplatelet Therapy Beyond 1 Year After Percutaneous Coronary Intervention. JAMA. 2016; 315(16): 1735-1749, doi: 10.1001/ jama.2016.3775, indexed in Pubmed: 27022822.

28. Costa F, van Klaveren D, James S, et al. Derivation and validation of the predicting bleeding complications in patients undergoing stent implantation and subsequent dual antiplatelet therapy (PRECISE-DAPT) score: a pooled analysis of individual-patient datasets from clinical trials. Lancet. 2017; 389(10073): 1025-1034, doi: 10.1016/S0140-6736(17)30397-5, indexed in Pubmed: 28290994.

29. Ndrepepa G, Neumann FJ, Schulz S, et al. Incidence and prognostic value of bleeding after percutaneous coronary intervention in patients older than 75 years of age. Catheter Cardiovasc Interv. 2014; 83(2): 182-189, doi: 10.1002/ccd.25189, indexed in Pubmed: 24030753.

30. Mori H, Cheng Qi, Lutter C, et al. Endothelial barrier protein expression in Biodegradable polymer sirolimus-eluting versus durable polymer everolimus-eluting metallic stents. JACC Cardiovasc Interv. 2017; 10(23): 2375-2387, doi: 10.1016/j. jcin.2017.06.059, indexed in Pubmed: 29102583. 